\title{
Preparation and Characterization of Liposomal Microencapsulated Poly- $\gamma$-glutamic Acid for Prevention of Ca-Phosphate Precipitation under Intestinal Environment
}

\author{
Hiroya ISHIKAwA, Yoshihiro SHImOdA and Kiyoshi Matsumoto \\ Laboratory of Food Analysis, Division of Food Biotechnology, Department of Bioscience and Biotechnology, Faculty of Agriculture, Graduate \\ School, Kyushu University, 6-10-1, Hakozaki, Higashi-ku, Fukuoka 812-8581, Japan
}

Received December 3, 2003; Accepted February 20, 2004

\begin{abstract}
Poly- $\gamma$-glutamic acid ( $\gamma$-PGA) from Bacillus subtilis (natto) with a high molecular weight (Av. M.W. $=350,000)$ effectively inhibited the formation of insoluble calcium phosphate at $\mathrm{Ca} / \mathrm{P}=0.5(12.5 \mathrm{~mm} / 25 \mathrm{mM})$ under the condition of $37^{\circ} \mathrm{C}$ and $\mathrm{pH}$ 7.4. Since decrease in inhibitory activity in $\gamma$-PGA was observed after the treatment by simulated gastric fluid ( $\mathrm{pH}$ 1.2, $0.5 \%$ pepsin), $\gamma$-PGA was encapsulated by egg lecithin liposome to keep the inhibitory activity under gastric conditions. Encapsulation was done by dehydration-rehydration (DR) and reverse-phase-vesicle (REV) methods. Higher encapsulation efficiency $(\mathbf{5 7 . 0 \%})$ was achieved by DR method. Turbidity measurement at $450 \mathrm{~nm}$ indicated the high stability of DR microcapsule, prepared with trehalose, against gastric juice. Encapsulated $\gamma$-PGA by DR method showed $\mathbf{9 3 . 3 \%}$ residual inhibitory activity after the gastric juice treatment.
\end{abstract}

Keywords: liposomes, microencapsulation, poly- $\gamma$-glutamic acid, Ca-solubilizing property

Intestinal calcium $(\mathrm{Ca})$ adsorption plays an important role in maintaining mineral homeostasis in human. Precipitation (insolubilization) of calcium phosphate $(\mathrm{CaP})$ decreases $\mathrm{Ca}$ absorption in the distal small intestine. Several studies have been made on the inhibition of $\mathrm{CaP}$ precipitation. Casein phosphopeptide (CPP) is well known to increase soluble Ca in small intestine. Lee et al. (1980) showed that casein phosphopeptides, which are formed during in vivo digestion of casein, well prevented calcium ion from CaP precipitation. Naito (1986) reported that CPP increase Ca solubility in vitro by preventing the formation of an insoluble complex of Ca with phosphate. Yamamoto et al. (1992) reported inhibitory activity of alginates, a dietary fiber, against the formation of CaP. They suggested that the interaction between the uronate residue of the alginate and calcium ion was important in the inhibition of $\mathrm{CaP}$ formation.

Poly-glutamic acids are one of the potent inhibitors against $\mathrm{CaP}$ precipitation in the distal small intestine. Naito (1986) reported that poly- $\alpha$-glutamic acid $(\alpha$-PGA) as well as CPP increased Ca solubility in vitro by inhibiting the formation of an insoluble CaP. The inhibitory effect of oligo- and poly-Lglutamic acids against $\mathrm{CaP}$ formation was investigated by Yamamoto et al. (1994). They showed that the inhibitory activity of oligo- and poly-L-glutamic acids would increase with their molecular weight. Poly- $\gamma$-glutamic acid ( $\gamma$-PGA), natural poly-anion peptides known as the main constituent of the sticky material of natto (fermented soybeans), has been expected for inhibitor of the $\mathrm{CaP}$ precipitation. Tanimoto et al. (2001) reported that calcium solubility in vitro and in vivo increased by the inhibition of $\mathrm{CaP}$ precipitation with natto mucilage containing $\gamma$-PGA. Tanimoto et al. (2003) have investigated the stimulatory effect of $\mathrm{Ca}$ supplement containing $\gamma$-PGA (Av.

E-mail: ishikawa@agr.kyushu-u.ac.jp
M.W. $=26,000)$ on $\mathrm{Ca}$ absorption by measuring urinary $\mathrm{Ca}$ excretion. They suggested that the Ca supplement containing $\gamma$ PGA increases Ca absorption in humans.

In this study, we investigated the inhibition of $\mathrm{CaP}$ formation by $\gamma$-PGA from Bacillus subtilis (natto) with a high molecular weight (Av. M.W.=350,000). The inhibitory effect of the $\gamma$ PGA in vitro was compared with those of $\alpha$-PGAs with various molecular weights. In addition, $\gamma$-PGA was encapsulated by microcapsule to reach the small intestine without degradation and/or aggregation by gastric juice. Liposomal microencapsulation is a method that enables intact biological material to reach the small intestine without affecting the quality of foods. We attempted to encapsulate $\gamma$-PGA by liposomal microcapsule by reverse-phase evaporation vesicle (REV) and dehydration-rehydration (DR) methods, which possess several advantages for encapsulation of valuable water-soluble materials, and to determine the effect of liposomal microencapsulated $\gamma$-PGA on the inhibition of insoluble $\mathrm{CaP}$ precipitation.

\section{Materials and Methods}

Materials $\gamma$-PGA from Bacillus subtilis (natto) (Av. M.W. $=350,000$ ) was donated by Dr. T. Hara of Kyushu University. $\gamma$-PGA, which is composed of D- and L-glutamate in the proportions of $6: 4$, is water-soluble and biodegradable. $\alpha$ PGAs (Av. M.W. =7,700, 14,300, 39,900, and 71,700) were purchased from Sigma Chemical Co. (St. Louis, MO, U.S.A.). Egg lecithin was purchased from Nacalai Tesque Co. (Kyoto, Japan). The bile salts, glycocholic acid, glycochenodeoxycholic acid, glycodeoxycholic acid, taurocholic acid, taurochenodeoxycholic acid, and taurodeoxycholic acid were purchased as sodium salts from Sigma Chemical Co. (St. Louis, MO, U.S.A.). Trehalose and all other chemicals were obtained from Nacalai Tesque Co. and were of analytical reagent grade.

Assay for the inhibitory activity of PGA on CaP precipita- 
tion in vitro Inhibitory effects of PGAs ( $\alpha$ - and $\gamma$-PGA) on the precipitation of insoluble $\mathrm{CaP}$ were assayed as follows. Each of PGA, $\mathrm{CaCl}_{2}$, and $\mathrm{Na}_{2} \mathrm{HPO}_{4}$ was dissolved in $50 \mathrm{mM}$ Tris- $\mathrm{HCl}$ buffer $(\mathrm{pH} 7.4)$. At the beginning of the reaction, $0.5 \mathrm{ml}$ of PGA solution (final conc. $0.5-2 \mathrm{mg} / \mathrm{ml}$ ) and $0.5 \mathrm{ml}$ of $50 \mathrm{mM}$ calcium chloride solution was mixed by vortexing. Then, $1.0 \mathrm{ml}$ of $50 \mathrm{mM} \mathrm{Na} \mathrm{HPO}_{4}$ solution was added $(\mathrm{Ca} / \mathrm{P}$ mol ratio $=1 / 2$ ), and the mixture was incubated at $37^{\circ} \mathrm{C}$. After incubation, precipitated $\mathrm{CaP}$ was removed by centrifugation at $3,000 \times \mathrm{g}$ for $5 \mathrm{~min}$. The concentration of calcium in the supernatant was determined by an $o$-cresolphthalein complexon method (Gitelman, 1967), of the Calcium C-test kit (Wako Pure Chemicals Co., Osaka, Japan).

Inhibition $(\%)$ of PGA was calculated by the following formula:

$$
\text { Inhibition }(\%)=\frac{C_{\mathrm{B}}-C_{\mathrm{C}}}{C_{\mathrm{A}}-C_{\mathrm{C}}} \times 100
$$

where $C_{\mathrm{A}}$ is initial $\mathrm{Ca}$ concentration, $C_{\mathrm{B}}$ is $\mathrm{Ca}$ concentration after the incubation for $30 \mathrm{~min}$ with PGA, and $C_{C}$ is Ca concentration after the incubation for 30 min without PGA.

Assay for the inhibitory activity of free and encapsulated PGA treated with simulated gastric fluid. Free and encapsulated PGA was suspended in $1 \mathrm{ml}$ of a $0.3 \%$ solution $(\mathrm{w} / \mathrm{v})$ of pepsin, dissolved in $\mathrm{HCl}$ solution containing $0.2 \% \mathrm{NaCl}(\mathrm{pH}$ 1.2). The suspension was incubated at $37^{\circ} \mathrm{C}$ for $4 \mathrm{~h}$, then was neutralized with $\mathrm{NaOH}$ to $\mathrm{pH} 7.4$. Inhibitory effects of free $\alpha$ and $\gamma$-PGA on $\mathrm{CaP}$ precipitation were assayed as described above. Inhibitory effect of encapsulated $\gamma$-PGA was similarly assayed after degradation of microcapsule by Hoffman bile acid solution (Hofmann, 1963). This solution, which simulated human intestinal content, was prepared by mixing of the six bile salts at the following ratio (by volume): sodium glycocholate, 30\%; sodium glycochenodeoxycholate, $30 \%$; sodium glycodeoxycholate, 15\%; sodium taurocholate, $10 \%$; sodium taurochenodeoxycholate, $10 \%$; sodium taurodeoxycholate, $5 \%$.

Residual inhibition $(\%)$ of PGA was calculated by the following formula:

$$
\text { Residual inhibition }(\%)=\frac{B}{A} \times 100
$$

where $A$ is the inhibition (\%) of intact PGA and $B$ is the inhibition $(\%)$ of PGA treated with simulated gastric fluid.

Liposomal microencapsulation of PGA Dehydrationrehydration (DR) microcapsules were prepared with trehalose according to the method described by Kim et al. (1999) with minor modifications. Egg lecithin in $150 \mathrm{ml}$ of ethanol was dried under reduced pressure at $37^{\circ} \mathrm{C}$ in a rotary evaporator to leave a thin film on the wall of a $500 \mathrm{ml}$ volume round-bottomed flask. The dried lipid film was suspended in $20 \mathrm{ml}$ of deionized water, and then sonicated by a bath-type sonicator for $10 \mathrm{~min}$ to produce small unilamellar vesicles (SUV). PGA was dissolved in $10 \mathrm{mM}$ sodium citrate solution and $10 \mathrm{ml}$ of the PGA solution was mixed with the SUV suspension. The mixture was then lyophilized. For rehydration, $1.5 \mathrm{ml}$ of $1 \%$ trehalose solution was added to the freeze-dried preparation and vortexed for $10 \mathrm{~min}$ (in encapsulation without trehalose, $1.5 \mathrm{ml}$ of deionized water was added for the rehydration.) The suspension was lyophilized again. The freeze-dried preparation was rehydrated by the addition of deionized water $(30 \mathrm{ml})$. DR microcapsules were separated from the suspension by centrifu- gation at $10,000 \times g$ for $10 \mathrm{~min}$. Unencapsulated PGA was hydrolyzed with $6 \mathrm{M} \mathrm{HCl}$ at $110^{\circ} \mathrm{C}$ for $20 \mathrm{~h}$, and then determined by high-performance liquid chromatography with 6-Naminoquinolyl- $N$-hydroxysuccinimidyl carbamate as described by Reverter et al. (1997). Encapsulation efficiency was calculated by the following formula:

Encapsulation efficiency $(\%)=$

$\{$ (weight of added PGA - weight of uncapsulated PGA)/ weight of added PGA $\} \times 100$

Reverse-phase evaporation vesicle (REV) method was done as described by Yoshimaru et al. (1999) with minor modification. The dried egg lecithin film was prepared by a method similar to that of DR method. After the dried film was dissolved in $50 \mathrm{ml}$ of ethanol, $10 \mathrm{ml}$ of the $\gamma$-PGA solution was added to the suspension. The resulting two-phase system was sonicated briefly $(1 \min \times 5)$ in a bath-type sonicator until the mixture became a clear one-phase dispersion. Ethanol in the mixture was removed under reduced pressure at $37^{\circ} \mathrm{C}$ in a rotary evaporator. The resultant REV microcapsules were separated from the suspension by centrifugation at $10,000 \times g$ for $10 \mathrm{~min}$. Similar to the above DR method, unencapsulated $\gamma$-PGA was determined and encapsulation efficiency was calculated.

Turbidity measurement of the microcapsule suspension Stability of the microcapsule against simulated gastric fluid ( $\mathrm{pH}$ 1.2) was investigated by turbidity measurement. The microcapsule was suspended in simulated gastric fluid at the initial turbidity of approximately 1.0, and then incubated at $37^{\circ} \mathrm{C}$ for $240 \mathrm{~min}$. The turbidity was measured spectrophotometrically at $450 \mathrm{~nm}$. The percentage of degradation in the microcapsule was calculated from the formula:

$$
\text { Degradation }(\%)=\frac{A-B}{A-C} \times 100
$$

where $A$ is the turbidity at $0 \mathrm{~min}, B$ is the turbidity during the treatment, and $C$ is the turbidity after complete degradation by treating with $25 \mathrm{~mm}$ Hofmann bile acid solution at $37^{\circ} \mathrm{C}$ for $60 \mathrm{~min}$.

Degradation of DR microcapsule by $10 \mathrm{~mm}$ Hofmann bile acid solution was monitored by the turbidity measurement in a similar manner. The percentage of degradation was calculated from the above formula.

Statistical analysis Results are presented as means \pm SD and analyzed using one-way analysis of variance followed by Tukey-Kramer test. All statistical analyses were performed using Stat View software version 5.0J (SAS institute Inc. USA).

\section{Results and Discussion}

Effect of PGA on CaP precipitation in vitro Inhibition of $\mathrm{CaP}$ precipitation at $\mathrm{pH} 7.4$ and $37^{\circ} \mathrm{C}$ with various amounts of $\gamma$-PGA is shown in Fig. 1 . When $\gamma$-PGA was not added, CaP precipitation was observed just after starting the incubation. Soluble Ca concentration decreased sharply to less than $1 \mathrm{~mm}$ within $5 \mathrm{~min}$. The $\mathrm{CaP}$ precipitation was inhibited by the addition of $\gamma$-PGA. Inhibitory effect of $\gamma$-PGA on $\mathrm{CaP}$ precipitation was shown at the concentration of above $0.5 \mathrm{mg} / \mathrm{ml}$ and soluble Ca concentration increased with increase in the concentration of $\gamma$-PGA. Little decrease in soluble $\mathrm{Ca}$ concentration was observed for $30 \mathrm{~min}$ at the $\gamma$-PGA concentration of $2.0 \mathrm{mg} / \mathrm{ml}$. In addition, the $\mathrm{Ca}$ concentration with $2.0 \mathrm{mg} / \mathrm{ml}$ of $\gamma$-PGA was kept at above $11 \mathrm{~mm}$ for at least $2 \mathrm{~h}$ (data not shown). 


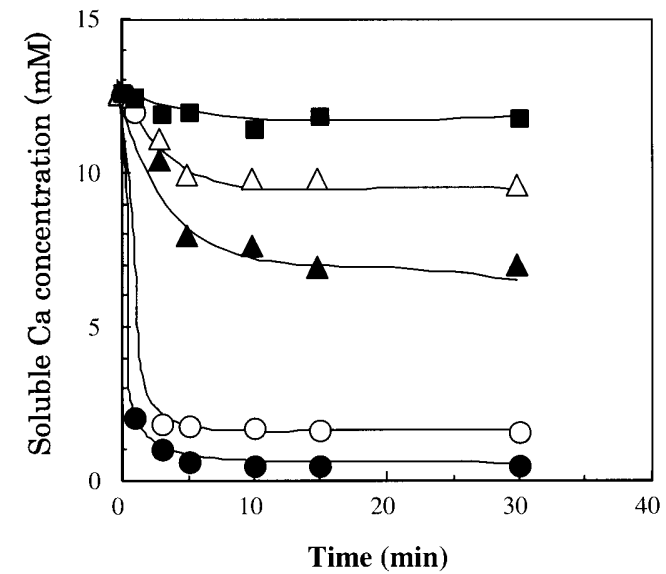

Fig. 1. Inhibition of calcium phosphate precipitation by addition of poly- $\gamma$ glutamic acid $\left(\gamma\right.$-PGA) at $\mathrm{pH} 7.4$ and $37^{\circ} \mathrm{C}$. $\gamma$-PGA was incubated with $12.5 \mathrm{mM} \mathrm{CaCl}_{2}$ and $25 \mathrm{mM} \mathrm{Na} 2 \mathrm{HPO} 4(\mathrm{Ca} / \mathrm{P}=0.5)$. Solid circles, $0 \mathrm{mg} / \mathrm{ml}$; open circles, $0.5 \mathrm{mg} / \mathrm{ml}$; solid triangles, $1.0 \mathrm{mg} / \mathrm{ml}$; open triangles, $1.5 \mathrm{mg} / \mathrm{ml}$; solid squares, $2.0 \mathrm{mg} / \mathrm{ml}$.

Table 1. Inhibition of calcium phosphate precipitation by $\alpha$ - and $\gamma$-PGA.

\begin{tabular}{ccc}
\hline Compounds & (Av. M.W.) & Inhibition $(\%)^{*}$ \\
\hline$\alpha$-PGA & $(7,700)$ & $21.2 \pm 3.32^{\mathrm{a}}$ \\
$\alpha$-PGA & $(14,300)$ & $29.4 \pm 5.18^{\mathrm{a}}$ \\
$\alpha$-PGA & $(39,900)$ & $55.4 \pm 8.83^{\mathrm{b}}$ \\
$\alpha$-PGA & $(71,700)$ & $75.7 \pm 5.75^{\mathrm{c}}$ \\
$\gamma$-PGA & $(350,000)$ & $93.0 \pm 2.10^{\mathrm{d}}$ \\
\hline
\end{tabular}

*Each assay was done by addition of PGA $(2 \mathrm{mg} / \mathrm{ml})$ at $\mathrm{pH} 7.4$ and $37^{\circ} \mathrm{C}$ for $30 \min (\mathrm{Ca} / \mathrm{P}=0.5)$ and values (mean $\pm \mathrm{SD}$ of 5 experiments) in a column sharing a common letter are not significantly different $(p>0.05)$.

These results suggest that $\gamma$-PGA would be able to effectively inhibit the Ca precipitation even at low $\mathrm{Ca} / \mathrm{P}$.

Inhibitory activity of natto mucilage containing $\gamma$-PGA on $\mathrm{CaP}$ precipitation was compared in vitro with that of $\alpha$-PGA (degree of polymerization $=535$ ) by Tanimoto et al. (2001). They showed that natto mucilage had lower activity than the $\alpha$ PGA. In this study, the inhibitory effect of $\gamma$-PGA in vitro was compared with that of $\alpha$-PGA with various molecular weights (Table 1). Average M.W. of $\alpha$-PGAs were 7,700, 14,300, 39,900 , and 71,700. All PGAs used in this study showed an inhibitory effect against $\mathrm{CaP}$ precipitation, and their inhibitory activity increased with their molecular weight. $\gamma$-PGA showed the highest inhibitory activity against the $\mathrm{CaP}$ precipitation (93\% inhibition) with activity over 15 percent higher than that of $\alpha$-PGA (Av. M.W. $=71,700$, degree of polymerization= 475). This result suggests that $\gamma$-PGA with high molecular mass in natto mucilage would have powerful inhibition against CaP precipitation. Yamamoto et al. (1994) investigated the effect of molecular weight on the calcium binding properties of oligo-L-glutamic acids (<20 mer: M.W. 2598) using a calcium-ion-selective electrode. They showed that the apparent amount of $\mathrm{Ca}$ bound to oligo-L-glutamic acids tended to increase with their molecular weight. In the present study, strong binding property of $\gamma$-PGA with high molecular mass to calcium-ion resulted in powerful inhibition against $\mathrm{CaP}$ precipitation.

Stability of PGA against simulated gastric juice To investigate the stability of $\alpha$ - and $\gamma$-PGA in the gastric environ-

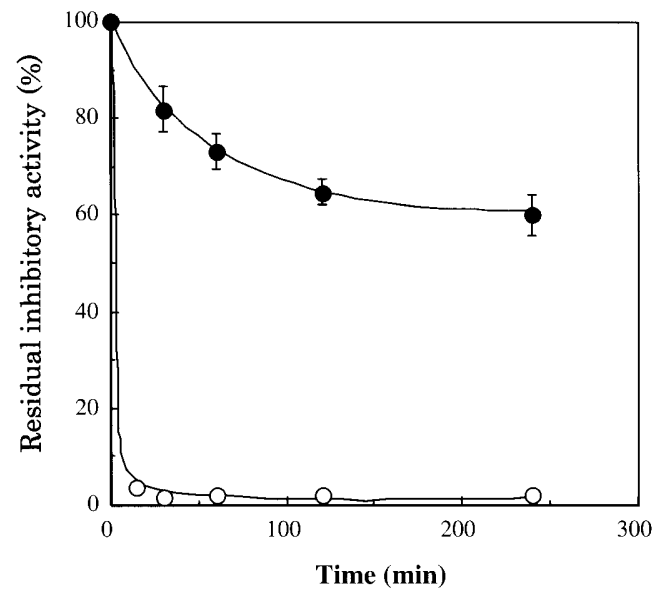

Fig. 2. Inhibition of calcium phosphate precipitation with $\alpha$ - and $\gamma$-PGA treated by simulated gastric solution at $37^{\circ} \mathrm{C}$. Each assay was done by addition of PGA $(2 \mathrm{mg} / \mathrm{ml})$. Each point represents the mean $\pm \mathrm{SD}(n=3)$. solid circles, $\gamma$ PGA; open circles, $\alpha$-PGA (Av. M.W. $=71,700$ ).

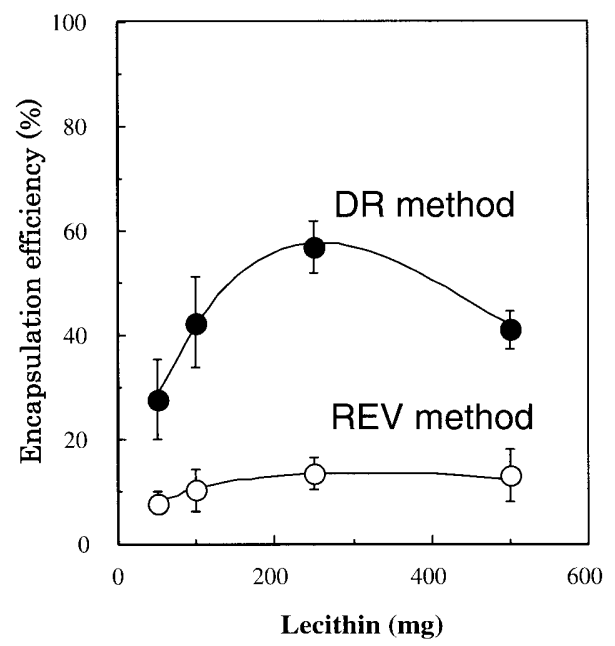

Fig. 3. Encapsulation of $\gamma$-PGA by dehydration-rehydration (DR) and reverse phase evaporation vesicle (REV) methods. Both encapsulations were done by using $25 \mathrm{mg}$ of $\gamma$-PGA. DR capsules were prepared with trehalose. Each point represents the mean $\pm \mathrm{SD}(n=3)$.

ment, each PGA was treated with a simulated gastric juice for $4 \mathrm{~h}$. Residual inhibitory activity of $\alpha$ - and $\gamma$-PGA on CaP precipitation was determined after this treatment (Fig. 2). The inhibitory activity of $\alpha$-PGA decreased to less than $5 \%$ within $15 \mathrm{~min}$; in contrast, slower decrease in the activity of $\gamma$-PGA was observed. Residual activity of $\gamma$-PGA was reduced to $60.1 \%$ after the gastric treatment for $4 \mathrm{~h}$. The difference in their tendencies would result from their molecular mass and/or the conformation in the gastric juice. In addition, only the gastric solution containing $\gamma$-PGA became turbid after $1 \mathrm{~h}$-incubation, and the turbidity increased with the passage of treatment time. In the absence of pepsin, similar increase in the turbidity was observed and the inhibitory activity of $\gamma$-PGA decreased to $83.1 \%$ after $4 \mathrm{~h}$ (data not shown). These results suggest that peptic degradation and/or aggregation of $\gamma$-PGA in the gastric environment would cause a great loss in the inhibitory activity of $\gamma$-PGA. 
Table 2. Encapsulation of Glu, Glu-Glu, $\alpha$-PGA, and $\gamma$-PGA by DR method.

\begin{tabular}{lcc}
\hline Compounds & (Av. M.W.) & Encapsulation efficiency $(\%)^{*}$ \\
\hline Glu & & $14.4 \pm 5.26^{\mathrm{t}}$ \\
Glu-Glu & & $30.9 \pm 7.17^{\mathrm{h}}$ \\
$\alpha$-PGA & $(7,700)$ & $58.8 \pm 4.61^{\mathrm{cd}}$ \\
$\alpha$-PGA & $(39,900)$ & $54.6 \pm 8.83^{\mathrm{c}}$ \\
$\alpha$-PGA & $(71,700)$ & $68.0 \pm 4.00^{\mathrm{d}}$ \\
$\gamma$-PGA & $(350,000)$ & $56.9 \pm 5.32^{\mathrm{cd}}$ \\
\hline
\end{tabular}

*Each capsule was prepared by using $25 \mathrm{mg}$ of glutamyl compound. Encapsulations were done by addition of trehalose. Values (mean \pm SD of 5 experiments) in a column sharing a common letter are not significantly different $(p>0.05)$.

Liposomal encapsulation of PGA To prevent loss in inhibitory activity of $\gamma$-PGA against $\mathrm{CaP}$ precipitation through a gastric environment, $\gamma$-PGA was encapsulated by egg lecithin liposomes. Encapsulation was done by DR and REV methods. In both methods, ethanol was used to prepare the liposome microcapsules. Other organic solvents, such as chloroform, diethyl ether, or methanol, carry a possible risk to human health and are unsuitable for industrial food use. Although the organic solvents are usually removed by evaporation, traces may be present in liposome formulation to cause a toxic problem. Figure 3 shows the encapsulation efficiency of $\gamma$-PGA by DR and REV methods with various amounts of egg lecithin. The encapsulation efficiency by DR method was higher than that by REV method. Highest encapsulation efficiency of $\gamma$-PGA $(25 \mathrm{mg}$ ) by the DR method was $57.0 \%$ using $250 \mathrm{mg}$ of egg lecithin.

Low encapsulation efficiency $(>15 \%)$ by the REV method would be due to the ethanol used to prepare the liposomes. Cortesi et al. (1999) prepared liposomes by REV method using ethanol and determined the encapsulation efficiencies of hydrophilic and hydrophobic model drugs. They suggested that encapsulation of hydrophilic compounds with ethanol would be difficult because hydrophilic compounds would not interact with the phospholipid bilayers formed in a water-ethanol system. In our encapsulation by the REV method, $\gamma$-PGA with hydrophilicity interacted little with egg lecithin bilayers in water-ethanol system.

Encapsulation of Glu, Glu-Glu, $\alpha$-PGAs, and $\gamma$-PGA were attempted by DR method (Table 2). Each DR microcapsule was prepared with $250 \mathrm{mg}$ of egg lecithin. High encapsulation efficiencies $(>50 \%)$ were achieved in all PGAs, and little difference was observed between $\alpha$-PGAs and $\gamma$-PGA. In contrast, encapsulation efficiencies of Glu and Glu-Glu were significantly low. It is likely that strong interaction between a number of carboxyl residues of PGA and hydrophilic groups of lecithin bilayers would result in high encapsulation efficiency in the DR method.

Resistance of DR microcapsules against gastric environment

To investigate the resistance of DR liposomes encapsulating $\gamma$-PGA against the gastric environment, turbidity measurement was done as described by Kim et al. (1999). Figure 4 shows the degradation ratios of DR liposomes calculated from the turbidity of liposome suspensions. To determine the effect of trehalose on the liposome stability in gastric solution, DR liposomes were prepared with and without trehalose $(15 \mathrm{mg} / 250 \mathrm{mg}-$ lecithin). These liposomes showed high stability against gastric solution. Stability of DR liposomes with trehalose was higher

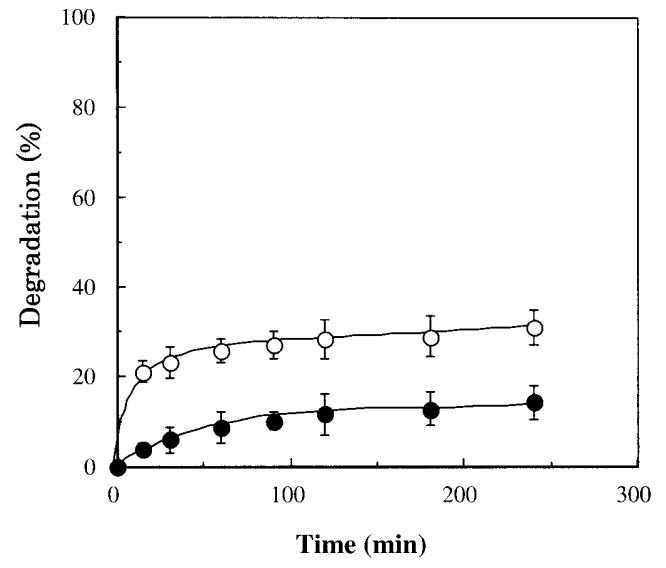

Fig. 4. Stability of DR microcapsules against simulated gastric juice at $37^{\circ} \mathrm{C}$ Stability of capsules was measured by turbidity monitoring at $450 \mathrm{~nm}$. DR microcapsules were prepared with (solid circles) and without (open cirlces) trehalose. Both encapsulations were done by using $25 \mathrm{mg}$ of $y$-PGA. Each point represents the mean $\pm \mathrm{SD}(n=3)$.

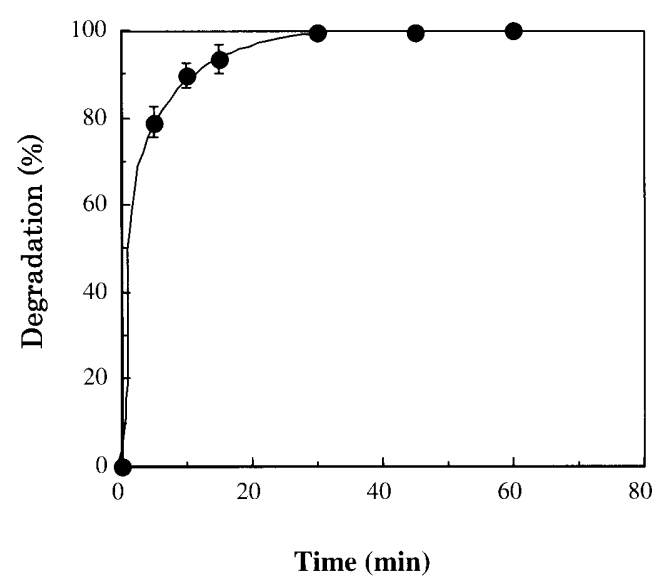

Fig. 5. Degradation of DR microcapsules with Hofmann bile acids (pH 6.4) at $37^{\circ} \mathrm{C}$. Degradation (\%) was measued by turbidity monitoring at $450 \mathrm{~nm}$. DR microcapsules were prepared with trehalose. Both encapsulations were done by using $25 \mathrm{mg}$ of $\gamma$-PGA. Each point represents the mean \pm SD $(n=3)$.

than that of the liposomes without trehalose. Degradation ratio of DR liposomes with trehalose was $14.2 \%$ even after gastric treatment for $4 \mathrm{~h}$. Similar results were obtained in the encapsulation of $\alpha$-PGA (data not shown).

Freeze-dried liposomes were stabilized by trehalose (Crowe et al.,1986). In the present encapsulation, therefore, trehalose would stabilize liposomes in a freeze-dried process in the DR method and result in high resistance of DR liposomes against the gastric environment.

Release of $\gamma$-PGA from DR liposomes by bile acids Release of $\gamma$-PGA from DR liposomes after passing through the stomach is required to inhibit $\mathrm{CaP}$ precipitation in the intestinal tract. Degradation of DR liposomes by bile acids, which is responsible for liposome digestion (Rowland \& Woodley, 1980; Nagata et al., 1990), was investigated by turbidity. Figure 5 shows the percent of degradation in DR liposomes prepared with trehalose. Rapid degradation was observed just after starting the incubation. Degradation ratio of DR liposomes reached 95\% within $15 \mathrm{~min}$. This result suggests that DR liposomes 


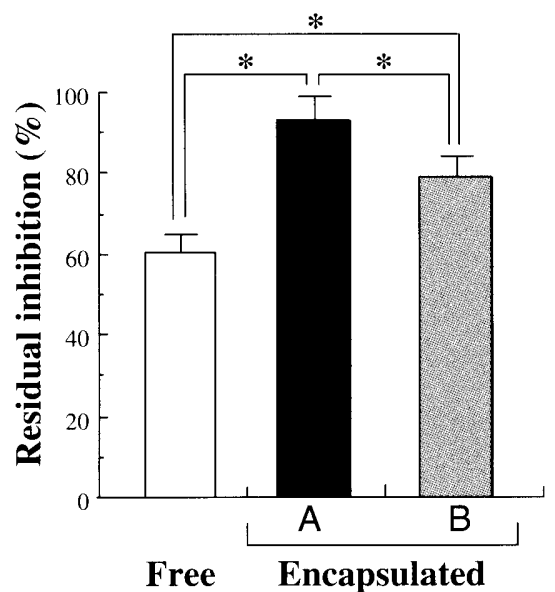

Fig. 6. Inhibtory effect on calcium phosphate precipitation with free and encapsulated $\gamma$-PGA treated by simulated gastric solution at $\mathrm{pH} 1.2$ and $37^{\circ} \mathrm{C}$ for $4 \mathrm{~h}$. Microcapsules were prepared by DR method with (A) and without (B) trehalose. Both encapsulations were done by using $25 \mathrm{mg}$ of $\gamma$-PGA. The results are expressed as means \pm SD of 6 experiments $(* p<0.001)$.

have an ability to release $\gamma$-PGA within their residence time in the bile salts-rich intestinal tract. In addition, DR liposomes encapsulating $\alpha$-PGA were easily degraded in a similar manner (data not shown).

Inhibition of CaP precipitation with encapsulated $\gamma$ $P G A \quad$ Inhibitory effect of free and encapsulated $\gamma$-PGA was determined after the treatment by gastric solution for $4 \mathrm{~h}$. Encapsulation was done by DR method with and without trehalose $(15 \mathrm{mg} / 250 \mathrm{mg}$-lecithin). After the gastric treatment, $\gamma$ PGA was released from DR capsules by the Hofmann bile acid solution, and then its inhibitory activity was assayed. Figure 6 shows residual inhibitory activities of free and encapsulated $\gamma$ PGAs on CaP precipitation after the treatment with gastric solution. DR liposomes were prepared with and without trehalose. As a result, protective effect of DR liposome against gastric treatment was observed. After the gastric treatment for $4 \mathrm{~h}$, 93.3 and $79.1 \%$ of residual inhibitory activities were obtained by encapsulated $\gamma$-PGA with and without trehalose, respectively. This suggests that $\gamma$-PGA encapsulated by DR method would pass through the stomach while retaining its inhibitory activity, and then, after being released from liposomes by the bile acids, inhibit the $\mathrm{CaP}$ precipitation in the intestinal tract.

\section{Conclusions}

The inhibitory effect of $\gamma$-PGA with high molecular weight on $\mathrm{CaP}$ precipitation was determined in vitro. Strong inhibitory effect of $\gamma$-PGA was shown at $\mathrm{Ca} / \mathrm{P}=0.5$, and a decrease in the inhibitory activity was observed after incubation in gastric solution with pepsin. To avoid the loss in the activity, $\gamma$-PGA was effectively encapsulated in liposomes by DR method. The liposome-microcapsules showed high resistance against gastric solution. Liposome-encapsulated $\gamma$-PGA showed $93.3 \%$ of intact inhibitory activity after the gastric juice treatment for $4 \mathrm{~h}$.

\section{References}

Cortesi, R., Esposito, E., Gambarin, S., Telloli, P., Menegatti. E. and Nastruzzi, C. (1999). Preparation of liposomes by reverse-phase evaporation using alternative organic solvents. J. Microencapsul., 16, 25I-256.

Crowe, L.M., Womersley, C., Crowe, J.H., Reid, D., Appel, L. and Rudolph, A. (1986). Prevention of fusion and leakage in freeze-dried liposomes by carbohydrates. Biochim. Biophys. Acta, 861, 131-140.

Gitelman, H.J. (1967). An improved automated procedure for the determination of calcium in biological specimens. Anal. Biochem., 18, 521531 .

Hofmann, A.F. (1963). The function of bile salts in fat absorption. J. Biochem., 89, 57-68.

Kim, C.K., Chung, H.S., Lee, M.K., Choi, L.N. and Kim, M.H. (1999). Development of dried liposomes containing $\beta$-galactosidase for the digestion of lactose in milk. Int. J. Pharm., 183, 185-193.

Lee, Y.S., Noguchi, T. and Naito, H. (1980). Phosphopeptides and soluble calcium in the small intestine of rats given a casein diet. Br. J. Nutr., 43, 457-467.

Nagata, M., Yotsuyanagi, T. and Ikeda, K. (1990). Bile salt-induced disintegration of egg phosphatidylcholine liposomes: A kinetic study based on turbidity changes. Chem. Pharm. Bull., 38, 1341-1344.

Naito, H. (1986). The mechanism of enhancement in intestinal calcium absorption with phosphate derived during casein digestion (in Japanese). J. Jpn. Soc. Nutr: Food Sci., 39, 433-439.

Reverter, M., Lundh, T. and Lindberg, J.E. (1997), Determination of free amino acids in pig plasma by precolumn derivatization with $6-\mathrm{N}$-aminoquinolyl- $N$-hydroxysuccinimidyl carbamate and high-performance liquid chromatography. J. Chromatogr: B., 696, 1-8.

Rowland, R.N. and Woodley, J.F. (1980). The stability of liposomes in vitro to $\mathrm{pH}$, bile salts and pancreatic lipase. Biochim. Biophys. Acta, 620, $400-409$.

Tanimoto, H., Nozawa, H., Okada, K. and Miyano, R. (2003). A calcium supplement containing poly- $\gamma$-glutamic acid increases human calcium absorption (in Japanese). Nippon Nougeikagaku Kaishi, 77, 504-507.

Tanimoto, H., Mori, M., Motoki, M., Torii, K., Kadowaki, M. and Noguchi, T. (2001). Natto mucilage containing poly- $\gamma$-gulutamic acid increases soluble calcium in the rat small intestine. Biosci. Biotechnol. Biochem., 65, 516-521.

Yamamoto, K., Kumagai, H., Sakiyama, T., Song, C.M. and Yano, T. (1992). Inhibitory activity of alginates against the formation of calcium phosphate. Biosci. Biotechnol. Biochem., 56, 90-93.

Yamamoto, K., Kumagai, H., Suzaki, A. and Arai, S., (1994). Inhibitory activity of oligo- and poly-L-glutamic acids against calcium phosphate insolubilization and calcium binding with special relevance to their molecular weight dependence. Biosci. Biotechnol. Biochem., 58, 1662 1665 .

Yoshimaru, T., Nakayama, M., Ohmachi, K. and Matsumoto,K. (1999). Preparation of liposomes for disintegration in the intestinal environment. J. Fac. Agric., Kyushu Univ., 43, 441-449. 